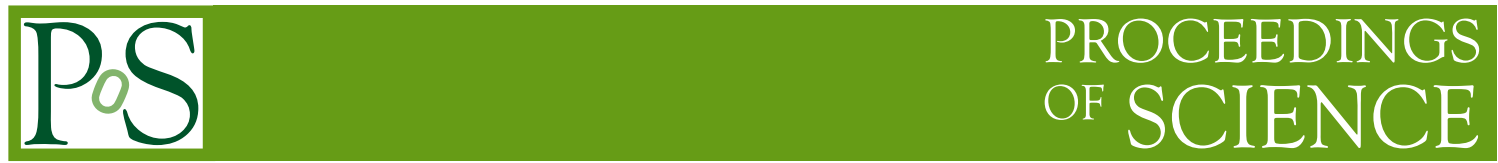

\title{
A High-Sensitivity Search for Charged Lepton Flavor Violation at Fermilab *
}

\section{E. Craig Dukes ${ }^{\dagger}$}

University of Virginia

E-mail: craigdukes@virginia.edu

The Mu2e collaboration proposes to search for coherent, neutrinoless conversion of muons into electrons in the field of a nucleus, $\mu^{-} N \rightarrow e^{-} N$, with a single-event sensitivity of $\sim 10^{-17}$, an improvement of a factor of 10,000 over existing limits. This lepton flavor-violating reaction probes mass scales unavailable by direct searches at either present or planned high energy colliders. The apparatus is briefly described as well as a scheme by which the experiment can be mounted in the present Fermilab accelerator complex. Prospects for increased sensitivity using the Project X accelerator that is being proposed by Fermilab are discussed.

10th International Workshop on Neutrino Factories, Super beams and Beta beams

June 30 - July 52008

Valencia, Spain

* On behalf of the Mu2e collaboration.

† Speaker. 


\section{Introduction}

The discovery of neutrino oscillations has shown that lepton flavor violation exists in the neutrino sector, and implies that charged lepton flavor violation should also exist, albeit at an unobservably low rate. There are many hints, however, both theoretical and experimental, suggesting that the standard model is incomplete. Many beyond-the-standard-model theories predict charged lepton flavor violation rates that could be seen in the next generation of lepton flavor violation experiments [1].

The Mu2e collaboration is proposing such a search at Fermilab in the coherent, neutrinoless conversion of a muon into an electron in the field of a nucleus, $\mu^{-} N \rightarrow e^{-} N$, a process which violates muon and electron number [2]. The proposed single-event sensitivity of $\sim 10^{-17}$ is 10,000 times that of the current best limit [3], and probes mass scales in excess of 3,000 TeV.

\section{Experimental Technique}

When a muon is stopped in a target it rapidly $\left(10^{-16} \mathrm{~s}\right)$ cascades down to the $1 \mathrm{~S}$ state. Several processes can then occur, the most likely being muon decay-in-orbit, $\mu^{-} N_{\mathrm{A}, \mathrm{Z}} \rightarrow e^{-} v_{\mu} \bar{v}_{e} N_{\mathrm{A}, \mathrm{Z}}$, and muon capture, $\mu^{-} N_{\mathrm{A}, \mathrm{Z}} \rightarrow v_{\mu} N_{\mathrm{A}, \mathrm{Z}-1}$, the former dominant for light nuclei and the latter dominant for heavy nuclei. Neutrinoless conversion of a muon into an electron, $\mu^{-} N_{\mathrm{A}, \mathrm{Z}} \rightarrow e^{-} N_{\mathrm{A}, \mathrm{Z}}$, results in an electron with an energy slightly less than that of the rest mass energy of the muon: $\sim 105 \mathrm{MeV}$. The major background to observing this process are electrons from muon decay-in-orbit, which unlike electrons from muon decay-in-flight, can recoil coherently off of the nucleus, resulting in endpoint energies up to that of the conversion electron energy. The tail of the muon decay-inorbit electron energy spectrum falls off as $\left(E-E_{e}\right)^{5}$ near the endpoint: less than $5 \times 10^{-15}$ of the electrons have an energy within $3 \mathrm{MeV}$ of the endpoint energy.

In order to achieve a sensitivity of $\sim 10^{-17}$, in excess of $10^{18}$ muons must be stopped; roughly $10^{11} \mathrm{~s}^{-1}$ ! A continuous muon beam, with its attendant pions and electrons, would produce unacceptably high detector rates and backgrounds. To mitigate this, in $M u 2 e$ the muon beam is bunched with a separation of roughly twice the captured muon lifetime of $864 \mathrm{~ns}$. The detector is turned off for some $700 \mathrm{~ns}$ after the intense bunched beam impacts stopping target because of the large flux of particles emanating from the target, and then turned on until the next bunch arrives. The experimental signature is an isolated $105 \mathrm{MeV}$ energy electron exiting the stopping target no less than $\sim 700 \mathrm{~ns}$ after the bunched beam has left.

\section{Producing the Bunched Muon Beam}

A scheme which takes advantage of upgrades to the Fermilab accelerator complex intended for the NOvA experiment and the availability of the Antiproton Accumulator and Debuncher rings after the termination of the collider program would be used to deliver protons to the Mu2e production target without affecting the neutrino program. It is illustrated in Fig. 1. The Booster accelerates proton batches to $8 \mathrm{GeV}$ every $1 / 15 \mathrm{~s}$. In a period of $1.33 \mathrm{~s} 12$ booster batches are momentum stacked in the Recycler ring and then transferred to the Main Injector where they are accelerated to $120 \mathrm{GeV}$ before being delivered to the NOvA neutrino production target. Since only 12 of a possible 20 Booster batches can be stacked during the $1.33 \mathrm{~s}$ Main Injector cycle, 8 Booster batches are 
available for other purposes. Six of them would be transferred (three at a time) via the Recycler ring from the Booster to the Accumulator where they would be stacked and then bunched into a single bunch with a period of $1.7 \mu \mathrm{s}$. The $2.4 \times 10^{13}$ protons would then be transferred to the Debuncher and slow extracted to a new experimental hall housing the $M u 2 e$ apparatus. A duty factor of up to 0.90 could be obtained.

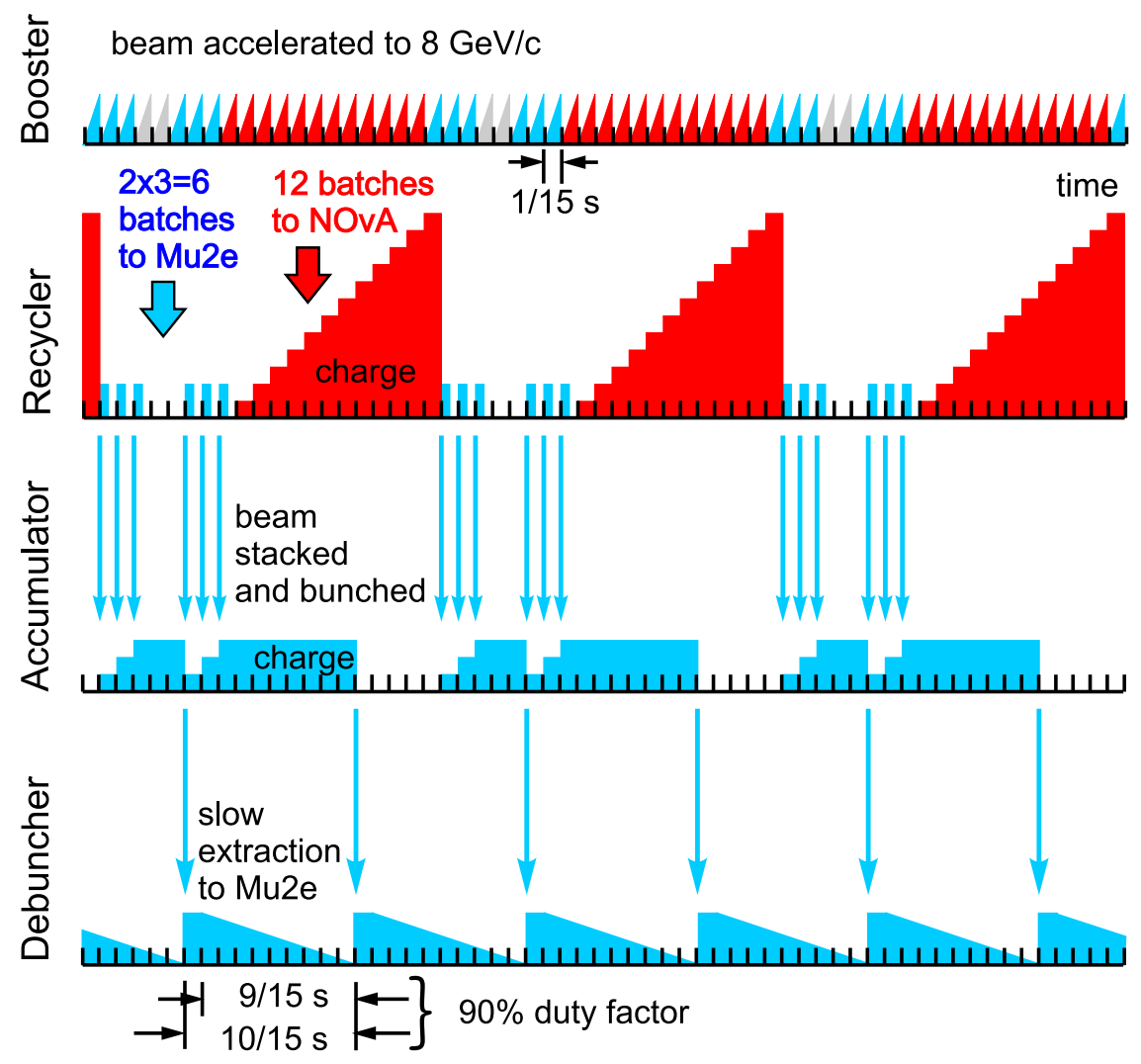

Figure 1: The $M u 2 e$ beam delivery scheme.

The $M u 2 e$ apparatus (Fig. 2) is identical to the MECO design proposed at Brookhaven [4]. It consists of three solenoidal magnets: the production, transport, and detector solenoids, with felds ranging from a high of $5.0 \mathrm{~T}$ at the far end of the production solenoid to $1.0 \mathrm{~T}$ at the opposite end of the detector solenoid. The bunched muon beam is produced as follows. The $8 \mathrm{GeV}$ proton bunches from the Debuncher ring enter the production solenoid at a slight angle to its axis and impact a water-cooled Au target. Pions are produced, which decay into muons, and some are captured in the graded field of the production solenoid and reverse direction, spiraling into the transport solenoid. The transport solenoid is curved in order to prevent line of sight transport of gammas and neutrons from the production target to the stopping target and to separate the positively and negatively charged particles. A collimator at the midpoint of the of the transport solenoid removes the positively charged particles. Approximately $0.25 \%$ of the protons impacting the production target produce muons that stop in one of the $170.2-\mathrm{mm}$ thick $\mathrm{Al}$ foils that form the stopping target.

Since the stopping target is in a graded magnetic field, particles emitted backward from the stopping target have their directions reversed. 


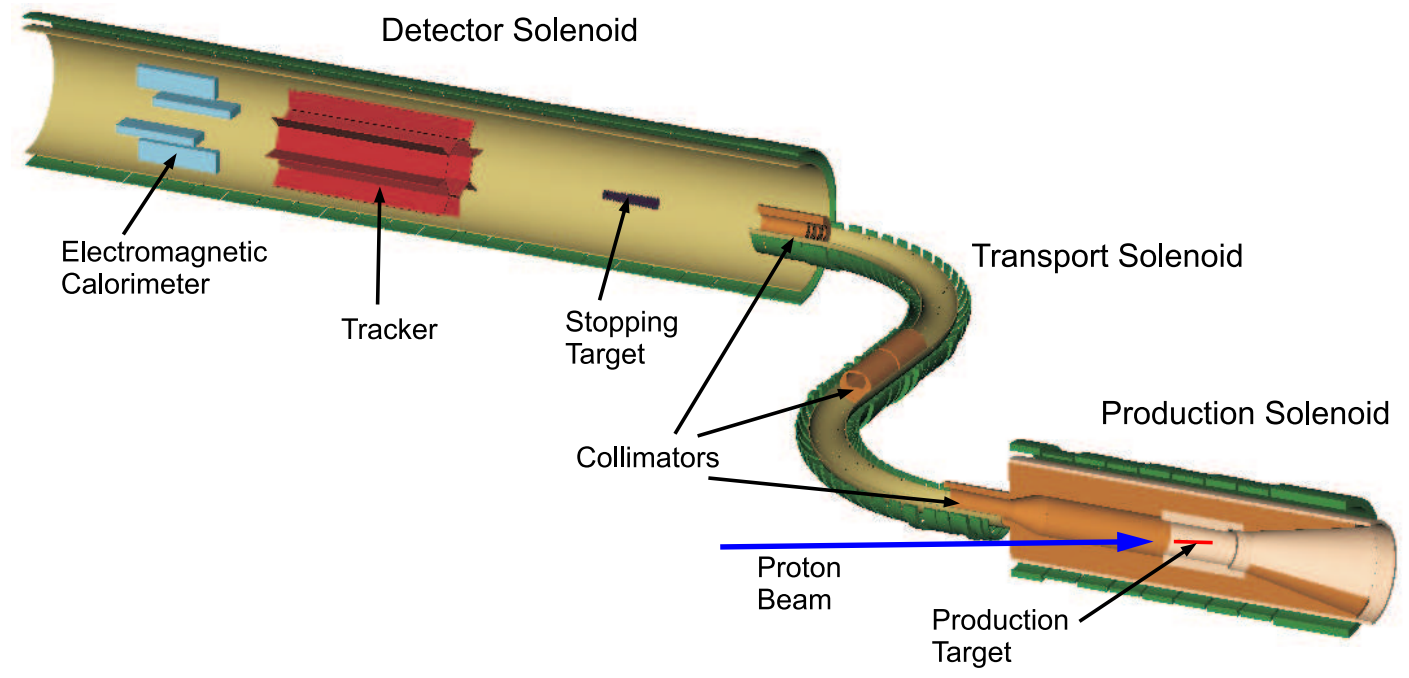

Figure 2: The Mu2e apparatus.

\section{The Detector}

The detector consists of a magnetic spectrometer, which is the primary energy measuring device, and an electromagnetic calorimeter, which is used for triggering and to confrm the energy and position measurements of the magnetic spectrometer. The Mu2e detector has been designed: (1) to ensure that the intense non-stopped beam from the production target is transported with minimal interactions to a beam stop at the end of the detector solenoid, (2) to minimize the acceptance for electrons from backgrounds such as muon decay-in-orbit, and (3) to have excellent momentum resolution for $\sim 100 \mathrm{MeV} / c$ electrons. The detector sits in an evacuated vessel in the $1 \mathrm{~T}$ field of the detector solenoid. It must withstand instantaneous rates up to $200 \mathrm{kHz}$ in individual detector elements.

The magnetic spectrometer consists of 2,800 $5 \mathrm{~mm}$ diameter straw tubes arranged in an octagon around the axis of the detector solenoid and in eight associated vanes. In addition there are 17,000 cathode strips that provide longitudinal position information. The momentum resolution is $\lesssim 0.200 \mathrm{MeV}$ and is dominated by multiple scattering. The geometrical acceptance of the magnetic spectrometer for conversion electrons is about $50 \%$.

Behind the magnetic spectrometer are $1,8003 \times 3 \times 12 \mathrm{~cm}^{3} \mathrm{PbWO}_{4}$ crystals arranged in four vanes. They are read out by avalanche photodiodes and provide an energy resolution of about $5 \%$ for electron energies of $100 \mathrm{MeV}$. A simple threshold on the calorimeter energy will be used to trigger on conversion electrons. Anticipated trigger rates are modest, as well as the data rate to permanent storage.

\section{Sensitivity and Backgrounds}

What is measured is the ratio of the muon to electron conversion rate to the muon capture rate: $R_{\mu e}=\Gamma(\mu N \rightarrow e N) / \Gamma\left(\mu N \rightarrow v_{\mu} N^{*}\right)$. In a run of $2 \times 10^{7}$ s a total of $3.6 \times 10^{20}$ protons would be incident on the production target. With a 0.61 capture probability, a 0.51 time-window acceptance, 
0.80 electron trigger efficiency, and a 0.19 reconstruction and selection efficiency, 4.3 events will be detected with $R_{\mu e}=10^{-16}$.

Backgrounds fall into three general categories: stopped muon induced backgrounds, beam related backgrounds, and time dependent backgrounds. The major background is estimated to be from muon decay-in-orbit. This background can only be defeated by good energy resolution. Beam related backgrounds are caused by interbunch protons and include beam electrons and electrons from muon decay-in-flight, $\mu^{-} \rightarrow e^{-} v_{\mu} \bar{v}_{e}$, and pion decay-in-flight, $\pi^{-} \rightarrow e^{-} \bar{v}_{e}$, which scatter in the stopping target foils, as well as electrons from radiative pion capture in the target, $\pi^{-} N_{\mathrm{A}, \mathrm{Z}} \rightarrow \gamma N_{\mathrm{A}, \mathrm{Z}-1}$, where the gamma converts into an asymmetric $e^{+} e^{-}$pair. These beam related backgrounds are defeated primarily by reducing the interbunch proton flux to less than $10^{-9}$ of the total. About $10^{-3}$ of this reduction comes from the normal RF bunching of the beam in the Accumulator and Debuncher, the remainder comes from special extinction systems. Cosmic rays are the only time dependent background: they are suppressed by active and passive shielding.

We estimate the total background number of events to be 0.4 , which would result in a signalto-noise ratio of 10 , assuming $R_{\mu e}=10^{-16}$.

\section{Mu2e in the Project X Era}

Recently Fermilab has proposed a substantial increase in proton intensity by replacing the $8 \mathrm{GeV}$ Booster with a linear accelerator with parameters similar to that proposed for the International Linear Collider (ILC). The new linear accelerator would produce seven proton batches in each 1.4 s-long Main Injector cycle, of which three would be injected into the Main Injector, leaving four for a high intensity $8 \mathrm{GeV}$ physics program. Use of all four would increase the $8 \mathrm{GeV}$ beam power to $M u 2 e$ from $20 \mathrm{~kW}$ to $200 \mathrm{~kW}$.

Although $M u 2 e$ has been designed to operate with a factor a three more beam power than the maximum provided by the Booster, going beyond this would require additional shielding in the production solenoid as well as increased production target cooling. The detector too would most likely have to be upgraded to handle the increased rates and to beat down the backgrounds in order to achieve a single-event sensitivity of $\sim 10^{-18}$. The experience gained in the "Booster era" running of $M u 2 e$ will undoubtedly prove crucial in determining what improvements need be made.

Despite these challenges, as well as many others that must be overcome, the prospect of achieving a single-event sensitivity of $\sim 10^{-18}$ is very exciting and will greatly expand our window into where new physics may appear.

\section{References}

[1] See, for example, Y. Kuno and Y. Okada, Rev. Mod. Phys. 73, 151 (2001).

[2] R.M. Carey et al., A Search for $\mu^{-} N \rightarrow e^{-} N$ with Sensitivity Below $10^{-16}$, FNAL Proposal 973, 2008.

[3] C. Dohmen et al., Phys. Lett. B317, 631 (1993).

[4] M. Bachman et al., A Search for $\mu^{-} N \rightarrow e^{-} N$ with Sensitivity Below $10^{-16}$, BNL AGS Proposal 940, 1997. 